\title{
METODOLOGÍA DE INVESTIGACIÓN DE LA RETÓRICA INTERCULTURAL APLICADA AL ELE
}

La retórica intercultural es una disciplina de la lingüística aplicada definida por Connor (2011) como "the study of written discourse between and among individuals with different cultural backgrounds" (p. 2). Este campo supone una redefinición de la más difundida retórica contrastiva, nacida a partir de las teorías de Kaplan (1966), quien estableció que los aprendientes de segundas lenguas transfieren los patrones retóricos y las convenciones culturales de su lengua materna a la lengua meta. La retórica contrastiva tenía ciertas carencias por las que fue constantemente criticada, lo que llevó a Connor (2004) a proponer el cambio de terminología. Para Connor (2011) es preferible el término intercultural debido a que "inter-" refleja la colaboración entre y dentro de las culturas y los individuos, mientras que "contra-" representaría oposición. Con ello, la autora, siguiendo nuevos avances dentro de la lingüística aplicada, pretende otorgar mayor importancia al contexto en el que se crean los textos, a las diferentes culturas (sean grandes o pequeñas) presentes en ellos y al proceso de negociación y acomodación que se da en la comunicación intercultural.

La retórica intercultural ha tenido una rica labor investigadora, especialmente en lo relacionado con el inglés como segunda lengua. Son numerosos los estudios llevados a cabo siguiendo los postulados de esta disciplina, lo cual ha desembocado en diferentes métodos de investigación. Sin embargo, por lo que respecta al español como lengua extranjera (en adelante, ELE), las investigaciones realizadas desde esta óptica han sido escasas, a pesar de presentar grandes posibilidades. El propósito de este trabajo es revisar los principales métodos de investigación empleados dentro de la retórica intercultural, así como sus posibles aplicaciones al ámbito del ELE, estableciendo futuras líneas de investigación dentro de este campo.

Connor (2011) afirma que las investigaciones de retórica intercultural siguen los patrones generales de los estudios llevados a cabo dentro de la lingüística aplicada. Así, el primer 
paso sería plantear la pregunta de investigación, o en su defecto el problema que pretende tratar y/o la hipótesis en la que se basa. Se continuaría con la recogida de datos y se finalizaría con su análisis e interpretación. Connor (2011), en colaboración con Moreno, ha desarrollado unas pautas de investigación en el marco del curso de doctorado "Research Methods in English/Spanish Crosscultural Studies" de la Universidad de León. Ambas expertas consideran que, una vez planeado el proyecto de investigación, conducido el estudio e informado sobre los resultados, es fundamental también realizarse a sí mismo unas determinadas preguntas respecto a si los objetivos del estudio se han cumplido y si se han explicado claramente los conceptos tratados y los resultados arrojados, entre otras cuestiones.

También establece Connor (2011) que los principales tipos de estudios llevados a cabo en retórica intercultural son el análisis de textos, la comparación de corpus con un determinado análisis lingüístico y las investigaciones etnográficas, lo que refleja que el análisis de textos ha centrado el foco investigador ya desde el nacimiento de la retórica contrastiva. En cualquier caso, los métodos de investigación de la retórica intercultural han superado los procedimientos de su predecesora gracias a las innovaciones en el ámbito general del estudio de la escritura, al entendimiento de la comunicación intercultural como un cúmulo de interacciones orales y escritas interrelacionadas y a la eclosión de los estudios sobre cultura.

El análisis de textos también se basa en la utilización de corpus, por lo que sus postulados investigadores son equivalentes a los de la comparación de corpus. Johansson (1998) reflejó los diferentes tipos de corpus empleados en las investigaciones entre culturas. En primer lugar, tenemos los corpus paralelos, formados por textos comparables en dos o más lenguas nativas. Otro tipo de corpus son los de traducción, compuestos por el texto original en su lengua nativa y su correspondiente traducción a una lengua extranjera. Finalmente, existen los corpus de aprendientes, que constan de producciones escritas realizadas en una segunda lengua. Moreno (2008) considera que en los corpus de aprendientes es necesario incluir textos comparables producidos por hablantes nativos. De estos tres tipos de corpus, Connor y Moreno (2005) establecen que los más utilizados en la retórica contrastiva han sido los corpus paralelos y los de aprendientes, si bien el estudio de la bibliografía al respecto nos permite afirmar que los corpus de traducciones también han sido ampliamente empleados.

Concidimos con Connor (2011) en que cada uno de estos tipos de corpus presenta unas determinadas ventajas para la investigación, siendo más útil alguno de ellos en función de nuestro objeto de análisis. Por un lado, los corpus paralelos permiten comparaciones reales a través de las culturas, mientras que los corpus de traducciones ayudan a estandarizar las traducciones y los corpus de aprendientes sirven para investigar el desarrollo de la interlengua del aprendiente. Así pues, tal y como Connor y Rozycki (2013) sugieren, "such corpora comparisons thus help teachers to understand reasons for potential mismatches in the formulation of specific text types by students" (p. 427). Además, en lo que al análisis de corpus se refiere, hay que tener en cuenta que en los últimos años, 
la informática ha comenzado a jugar un papel crucial en su procesamiento. Actualmente, en contraste con la recogida manual de los orígenes, la inmensa mayoría de los corpus son informatizados, por lo que, como Pérez-Llantada (2008) afirma, "it seems that machines (corpora) and humans (researchers) need to work hand in hand when identifying rethorical differences across cultures" (p. 92). Connor (2013) también aboga por la utilización de los ordenadores. En relación con ello, Connor y Rozycki (2013) afirman que

with the remarkable advances in information technology over the past few decades, corpora can now easily be digitally collected, manipulated and statistically analized. This allows for cross-comparison of far more specimens of text, lending greater power to findings in intercultural rhetoric research (p. 434)

Por ejemplo, un programa informático que sirve para estos análisis es WordSmith Tools, empleado por Balažic Bulc (2005). En su investigación, la autora comparaba el uso de conectores por parte de universitarios eslovenos y croatas en varios géneros textuales tanto en lengua materna como extranjera. El programa informático WordSmith Tools, en su versión 3.0, le sirvió para localizar y analizar cuantitavimante los conectores empleados en los textos ${ }^{1}$.

En todos estos tipos de análisis un concepto clave es el de tertium comparationis, introducido a la retórica contrastiva por Enkvist (1997) y ampliamente tratado por Connor y Moreno (2005) y Moreno (2008). Enkvist (1997) estableció que "tertium comparationis is a concept, or a set of concepts, that can be used as a basis for comparison" (p. 196). Connor y Moreno (2005) aclaran que el concepto de tertium comparationis "is important at all levels of research: in identifying texts for corpora, selecting textual concepts to be studied in the corpora, and identifying linguistic features that are used to realize these concepts" (p. 155). Por su parte, Moreno (2008) considera necesario contrastar cosas comparables entre las culturas, equiparando el concepto de comparabilidad al de equivalencia. A este respecto, aclara que, para que dos corpus sean comparables, no se necesita que sean exactamente iguales, sino similares al máximo grado. Un problema relacionado con esto que la autora menciona es que la similitud de los textos depende mucho de quién los interprete. Moreno (2008) establece que los escritos serán comparables si todos los factores contextuales incluidos en el texto pueden ser considerados similares. Estos factores pueden comprender el género y el tema del texto, su estructura global, el espacio físico y temporal en el que se escribe, los textos previos a la versión final realizados, el grado de pericia del autor, el estado emocional de los escritores, sus necesidades comunicativas (probablemente el factor más relevante), el modo y el canal de comunicación y la motivación y la personalidad del autor, entre otros. Los factores sexuales y de edad pueden también tener su importancia. Otro aspecto que debe ser equiparable es el de la

1 Para profundizar en la utilización de corpus en la retórica intercultural es de obligada consulta el trabajo de Belcher y Nelson (2013). 
longitud y la proporción de textos de uno y otro corpus. Así, Connor y Moreno (2005) proponen que los corpus no deben ser solo similares en términos estructurales y funcionales, sino también estadísticos.

Moreno (2008) plantea que en los estudios contrastivos los corpus deben ser comparables en todos los factores contextuales excepto en el código lingüístico (la cultura escritora a la que pertenece el autor, es decir, su idioma materno) y la forma y el contenido del texto (aspectos lingüísticos, semánticos y retóricos), ya que estos representan las variables independiente y dependiente de la investigación, respectivamente. En cuanto a las variables que se quieren analizar, Connor y Moreno (2005) aclaran que

it must be stressed that variables should be formulated according to a common theoretical framework that provides appropriate objective tools for analyzing textual material from the two languages. This is a difficult stage in contrastive rhetoric research since the descriptions of similar phenomena available in the two languages are likely to have used different theoretical frameworks, and may not be compatible. So, the task of the researcher is to choose or design tailor-made tools to apply to the analysis of the two text-rethorical systems independently (p. 9)

Esto significa que el tertium comparationis debería basarse más en significados funcionales que en criterios formales. En este sentido se pronuncia también Enkvist (1997), quien se cuestiona hasta qué grado es posible realizar comparaciones generales y específicas de base cultural entre las lenguas debido a la elasticidad del término "cultura", lo que imposibilita presentar una lista completa de dichos aspectos. Otros factores contextuales, como el sexo, serían variables intervinientes. Moreno (2008) afirma que en los estudios descriptivos no es posible manipular las variables, por lo que el diseño del corpus debe ser extremadamente cuidadoso con el fin de controlar estadísticamente todas las variables intervinientes. En el caso de que se controlen y mantengan constantes, se podrá afirmar que los corpus son equivalentes al máximo grado, dando lugar a comparaciones y resultados válidos. No obstante, hay factores concretos que son imposibles de controlar, como la propia idiosincrasia del escritor, entre otros. Por tanto, en lo que respecta a determinados puntos, los corpus nunca serán comparables.

Teniendo en cuenta todo esto, Connor y Moreno (2005) establecen un programa de doce pasos para llevar a cabo una investigación de retórica intercultural con corpus, que se enumeran a continuación: 1. Formular hipótesis acerca de las relaciones entre las culturas escritoras; 2. Definir la población de autores comparables; 3. Seleccionar una muestra representativa de esos autores; 4. Identificar unidades textuales o lingüísticas comparables; 5. Validar esas unidades a través de investigación secundaria; 6. Cuantificar la aparición de esas unidades en los corpus; 7. Diseñar criterios específicos para la realización de esas unidades en ambas lenguas; 8. Aplicar esos criterios a los dos corpus independientemente; 9. Yuxtaponer las taxonomías; 10. Contrastar los resultados cuantitativos; 11. Interpretar 
las diferencias y similitudes a través de análisis estadístico; y 12. Sacar conclusiones sobre las relaciones entre las culturas escritoras.

A pesar de los avances en lo referente a la comparabilidad de los corpus, Moreno (2008) opina que "considerable work still needs to be done to assure that the corpora are really comparable" (p. 32). Así, sigue siendo difícil elegir textos equivalentes. En este sentido, Connor (2011) advierte de que, además, "in establishing tertium comparationis we have to be careful not to essentialize discourse communities or belittle individual variation in the production and reception of the genres studied" (p. 50) ${ }^{2}$. Esta misma autora sugiere que es muy difícil incluir el análisis del contexto de escritura en las investigaciones, las cuales tienen como principal reto encontrar el equilibrio entre el tratamiento cuantitativo y el cualitativo. En cualquier caso, tal y como afirman Connor y Moreno (2005), los trabajos que siguen el tertium comparationis son eminentemente de carácter cuantitativo.

Uno de los ámbitos en los que se ha desarrollado enormemente el análisis de textos y la comparación de corpus es el relacionado con los géneros discursivos. Según Connor (2011), el auge del análisis de géneros ha sido extremadamente beneficioso para la retórica intercultural, ya que ha obligado a los investigadores a comparar textos equivalentes. En este sentido también se pronuncian Connor y Rozycki (2013), para quienes "genre analysis has provided researchers with methods of analysis that supplement the discourse analysis methods used in previous contrastive rhetoric research" (p. 431). Estos especialistas recuerdan que la comparación de géneros a través de culturas no puede caer en la creación de estereotipos de las comunidades discursivas ni ignorar la variación individual en los textos. Con este fin, estudios recientes como el de Escamilla (2013) introducen el análisis crítico del discurso para examinar de manera argumentada las prácticas sociales que rodean a la creación de textos.

Por último, uno de los métodos de investigación más prometedores de la retórica intercultural es el etnográfico. A pesar de las posibilidades que ofrece, los estudios que han seguido este enfoque han sido escasos, incluso si fueron reclamados en el pasado por Kaplan y Grabe (1996) como una de las prioridades de la disciplina. Este modelo de investigación se centra especialmente en los factores contextuales que rodean a la escritura y que mencionábamos más arriba. En este método el investigador se convierte en participante y observador y se centra en el análisis de un determinado aspecto (por ejemplo, la forma de interiorizar un determinado género discursivo en el sistema educativo de una cultura o la creación o no de borradores dentro de una comunidad discursiva ${ }^{3}$ ). Connor (2011) estipula que, como participante, el investigador no analiza nada, todo lo contrario que realiza como observador, cuando analiza absolutamente todo. Para llevar a cabo su labor puede valerse de entrevistas, grabaciones y/o observaciones de clase, notas de campo, grupos de discusión, etc. Debido a sus propias características, se trata de una investigación de corte transversal y diacrónico, si bien Connor (2011) afirma que en la retórica intercultural no se realizan investigaciones

2 Cursiva en el original.

3 Los ejemplos son nuestros. 
etnográficas realmente intensivas y a largo plazo, sino que se limitan a emplear algunas de sus técnicas. En cualquier caso, esta autora concluye que "these methods can add richness and accuracy to the interpretation. The approach can be called semi-etnographic" (p. 61). Debemos hacer notar, coincidiendo con Enkvist (1997), que este tipo de estudio presenta el problema de la observación subjetiva del propio investigador, el cual siempre en mayor o menor grado está delimitado por sus propias concepciones culturales, pudiendo pasar por alto diferencias o similitudes entre las culturas analizadas.

\section{APLICACIONES AL ELE}

La retórica intercultural es una disciplina que nació en un contexto anglosajón, de ahí que sus fundamentos teóricos y las investigaciones realizadas hayan girado mayoritariamente en torno al inglés como segunda lengua. De hecho, la propia Connor (2011) afirma que entre las piedras angulares del campo está el inglés con fines específicos. Por consiguiente, los trabajos que siguen este enfoque relacionados con el ELE son escasos, tal y como se muestra en Heredero Zorzo (en prensa). No obstante, el español ha sido una de las lenguas más empleadas en el contraste con el inglés, por lo que, si bien no suponen investigaciones de ELE, muchas de sus conclusiones pueden resultar de gran interés para los profesionales de este campo. En esta parte de nuestro trabajo presentamos brevemente algunos de los estudios llevados a cabo, bien propiamente de ELE, bien que incluyan el español aplicado al inglés como segunda lengua, que emplean los métodos de investigación presentados en el capítulo anterior. El objetivo es mostrar cómo es posible aplicar la metodología de investigación de la retórica intercultural al ELE para, finalmente, establecer las líneas de investigación abiertas al respecto.

Como ya hemos indicado más arriba, el principal método de investigación de la disciplina es el análisis de textos a través de la comparación de corpus paralelos. Coincidimos con Connor y Moreno (2005) en que este tipo de investigación es necesaria "if there is no previous empirical research or proven theory concerning similarities and differences in linguistic/rhetorical structures in a given genre between the two languages and cultures" (p. 12). Un ejemplo de este trabajo es el de Suárez y Moreno (2008), quienes emplean corpus de este tipo para analizar y contrastar las características del género de la reseña académica en inglés y español. Concretamente, estudian los movimientos del texto y las subfunciones incluidas en ellos. En este trabajo se llega a la conclusión de que ambas culturas comparten numerosas estructuras retóricas, si bien los hablantes españoles prefieren más los movimientos descriptivos y critican de manera abierta mucho menos el libro reseñado. Así pues, esta investigación podría servir como ejemplo para futuros trabajos centrados en el uso de corpus paralelos y el análisis de géneros discursivos.

En cuanto a los corpus de aprendientes, por razones obvias este es el tipo de datos más empleado en las investigaciones aplicadas al ELE. Como muestra de ello podemos 
mencionar los trabajos de Deza Blanco (2007) y Heredero Zorzo (2016), quienes contrastan el uso de marcadores discursivos en español entre hablantes nativos y no nativos. El primero lo hace en noticias de sucesos escritas por periodistas españoles y aprendientes taiwaneses de ELE, mientras que el segundo se basa en ensayos expositivo-argumentativos redactados por universitarios españoles y eslovenos. Llama la atención que ambos trabajos presentan deficiencias en lo que al tertium comparationis se refiere. Deza Blanco (2007) no respeta todos los factores contextuales, ya que el grado de pericia de los autores no es el mismo, al comparar textos escritos por profesionales con otros producidos por universitarios. Por su parte, las producciones escritas en las muestras recogidas por Heredero Zorzo (2016) no se realizaron en el mismo contexto espaciotemporal, puesto que los aprendientes eslovenos de ELE tuvieron dos semanas para escribir en casa y los hablantes nativos tan solo una sesión de clase.

Coincidimos con Connor y Moreno (2005) en que, tanto los corpus paralelos como los corpus de aprendientes, son herramientas imprescindibles a la hora de describir y explicar las similitudes y las diferencias existentes entre culturas en relación a aspectos textuales y retóricos. Así, las investigaciones basadas en corpus paralelos servirían para conocer determinados aspectos de lenguas nativas que podrían resultar de enorme interés para profesores de ELE con estudiantes procedentes de esas otras culturas. Por otro lado, los corpus de aprendientes mostrarían cuáles son las similitudes y las diferencias en el uso de patrones y estructuras concretas entre hablantes nativos y no nativos, proporcionando a los docentes una valiosa información para aplicar a sus clases. En lo referente a los corpus de traducciones, cuya utilidad al mundo del ELE quizás podría reducirse a la enseñanza de estudiantes de Traducción, se pueden consultar diferentes investigaciones en Penas Ibáñez y Martín Martín (2009).

Previamente hemos hablado de la importancia del tertium comparationis para tener resultados válidos en las investigaciones. Si se quiere consultar un trabajo que sigue todos sus postulados, es de obligada consulta el trabajo de Moreno (1998), en el cual se compara la señalización de las premisas y las conclusiones en artículos de investigación en inglés y español. De hecho, es a partir de este estudio del cual se establecieron las características del tertium comparationis desarrolladas en Connor y Moreno (2005) y Moreno (2008). Si se toma este trabajo como modelo, los resultados del análisis serán siempre válidos y sus conclusiones firmes.

Más arriba se ha visto que los estudios de carácter etnográfico no han tenido un amplio recorrido, mucho menos si de lo que tratamos es del ELE. Un estudio digno de mención por su profundidad, y que tiene el español entre una de sus lenguas, es el llevado a cabo por LoCastro (2008). Esta autora analiza la escritura de universitarios mexicanos en inglés, incluyendo en el estudio, además de los textos producidos por los estudiantes, observaciones de clase, entrevistas con profesores e informantes, varios documentos relacionados con el sistema educativo y datos de tutorías con participantes en el proyecto de investigación. De cierta similitud con un tratamiento etnográfico es el trabajo de Jiménez 
Ramírez (2010), quien puso en práctica una aplicación pedagógica trabajando sobre los fundamentos de la retórica contrastiva para mejorar la escritura de aprendientes de ELE de diferentes orígenes. Por un lado, las investigaciones etnográficas proporcionarían información sobre los factores contextuales del proceso de escritura, lo que resultaría de gran interés para los profesores de ELE, tratándose además de un tipo de datos a los que habitualmente no se tiene acceso. Por su parte, estudios centrados en tratamientos pedagógicos aportarían conocimientos sobre cómo el empleo de las bases teóricas de la retórica intercultural afecta a la escritura de los aprendientes.

De la corta trayectoria de los estudios de retórica intercultural aplicados al ELE podemos deducir que las líneas de investigación que deja abiertas son prácticamente infinitas. Estas líneas de investigación no han sido establecidas hasta la fecha para esta segunda lengua, aunque sí para el inglés. Así, Connor (2011) menciona que en el caso del inglés como segunda lengua los futuros estudios deberían centrarse en aspectos como las negociaciones económicas internacionales, la traducción y la interpretación y la formación de personal médico y de trabajadores inmigrantes. Esta autora también propone como un campo con interesantes perspectivas el análisis de la retórica visual multimodal, lo que Beck (2011) define como la integración de estímulos visuales en los textos. A este respecto, Connor (2011) concluye que "for intercultural rhetoric to continue as a viable area of research with practical implications, it has to be mindful of the powerful interactions of oral, written, and multi-modal discourses in message formulation in intercultural communication" (p. 54). Otra dirección interesante que propone esta investigadora se refiere a la inclusión de los procesos de negociación y acomodación que se dan en la retórica intercultural en las pruebas de certificación lingüística. En relación con ello, sugiere que "there still seems to be a lack of sensitivity to cultural variation" (p. 88). En otro trabajo, Connor y Rozycki (2013) abogan por un mayor análisis de los géneros, tales como las propuestas de comunicaciones en congresos, sus presentaciones en los congresos y las solicitudes de becas de investigación. Estos dos autores también proponen el estudio de los contextos y los procesos de escritura (incluyendo el estudio de los borradores, la correspondencia entre editores y revisores y las negociaciones entre coautores), ya que "this type of granular ethnographic research is the best way to identify the cultural differences that impede the success of academic writers in an L2 setting" (p. 440).

Gran parte de estas líneas de investigación abiertas para el inglés como segunda lengua son compatibles con el ELE, aunque no todas, como es el caso de dos de los grandes focos de la retórica intercultural actual: el inglés como lengua franca y la formación de personal médico. Lo primero se debe a que el español tiene poco recorrido como lengua franca, mientras que en los países de habla hispana no hay una presencia relevante de trabajadores del sector médico que no sean hablantes nativos. Por el contrario, consideramos que sí es de gran interés para el ELE la investigación sobre el lenguaje académico. El análisis del inglés académico entre hablantes no nativos ha llevado el peso de la retórica contrastiva/intercultural en la última década del siglo pasado y en la primera del 
actual. Así, numerosas investigaciones se han llevado a cabo contrastando la escritura de académicos no nativos en inglés y en sus idiomas maternos. Dentro de ellos, son muchos los trabajos realizados al respecto que incluyen el español como una de las lenguas de contraste, de los cuales podemos citar los de Martín Martín (2005) y Mur-Dueñas (2016) como ejemplos. Sería de gran interés tomar los resultados de estos estudios y contrastarlos con trabajos académicos publicados en español por hablantes no nativos (y también en sus respectivas lenguas maternas), con el fin de establecer conclusiones respecto a los patrones retóricos y culturales de la escritura de estos últimos. Un ejemplo concreto de investigación podría ser tomar artículos de investigación de lingüística aplicada escritos por hablantes nativos y por hablantes no nativos de una cultura de origen concreta escritos en ELE y en su lengua materna, para a partir de ello analizar diferentes factores, como los diferentes movimientos del texto y sus subfunciones, y las estructuras preferidas en ellos. También se podrían analizar las propuestas de comunicaciones a congresos, como sugerían Connor y Rozycki (2013). Opinamos que estudios de estas características serían muy relevantes para el campo del ELE, puesto que son numerosos los especialistas no nativos que se dedican a publicar en revistas especializadas y a participar en los diversos congresos que se organizan. Además, las perspectivas de investigación son amplias, ya que el contraste se podría realizar con tantas otras lenguas como profesionales procedentes de esas culturas haya.

Esta línea de investigación nos lleva a otra similar como es el análisis de géneros. Respecto a esto también hay muchos trabajos que contrastan géneros discursivos en español con el inglés, por lo que también estas publicaciones podrían servir como punto de partida. Es más, las investigaciones se podrían abrir a cualquier género que no haya sido tratado con anterioridad, planteando así también numerosas posibilidades investigadoras. Como ejemplo, debido al aumento en los últimos años del número de estudiantes con otra lengua materna y procedentes de otras culturas en las universidades de España, proponemos el análisis de determinados géneros que tienen presencia en estas situaciones, como podrían ser las solicitudes de admisión en un máster o doctorado o una solicitud de beca de estudios. Se trata de géneros discursivos no analizados hasta el momento, por lo que sus resultados podrían ser de enorme ayuda a la gran cantidad de aprendientes de ELE que se encuentran en esas situaciones. Aquí las posibilidades de investigación serían también tan numerosas como las diferentes procedencias culturales de los estudiantes.

Además, las investigaciones centradas en el español como lengua académica y las de análisis de géneros, podrían ir acompañadas de estudios etnográficos al respecto, si no de manera profunda, al menos con determinadas herramientas. Así, en trabajos sobre el español de académicos no nativos sería interesante analizar cómo se enseña la escritura académica en sus culturas de origen, cuáles son los requisitos para la publicación en las revistas especializadas de sus países o si sus textos pasan por correctores ortográficos o solicitan algún otro tipo de ayuda a la hora de redactarlos. Por su parte, en el caso de los estudiantes universitarios de ELE, se realizarían investigaciones similares, observando si es necesario 
en su cultura de origen, por ejemplo, redactar una carta de solicitud de admisión para unos estudios concretos y si se les enseña a cómo hacerlo. También se podría estudiar cómo afrontan los alumnos esta tarea, teniendo entrevistas personales con ellos para cuestionarles acerca de cuánto tiempo le dedican a su escritura, en qué contexto espacial la llevan a cabo, si realizan borradores, si consultan ejemplos de este tipo de texto, etc.

A un nivel de pericia menor también se presentan muchas líneas de investigación abiertas para el ELE en cuanto al método etnográfico se refiere. Los estudiantes de enseñanza secundaria y universitaria de grado tienen que hacer frente con frecuencia a la redacción de ensayos, entre otros tipos de texto. Hemos visto más arriba que son muchas las investigaciones que se dedican a analizar la escritura de aprendientes de ELE de estos géneros discursivos, como en el caso de Heredero Zorzo (2016). Sin embargo, son muy pocos los trabajos que incluyen una parte etnográfica que pretenda explicar a qué se deben las similitudes y diferencias entre la escritura de los hablantes nativos y no nativos, siendo una excepción el estudio de LoCastro (2008). Consideramos que, para progresar en el entendimiento de por qué los aprendientes de ELE producen textos de una determinada manera, se debe investigar mediante técnicas etnográficas, realizando observaciones de clases en las que se trabaje la enseñanza de la escritura de esos géneros discursivos, manteniendo entrevistas con profesores y alumnos sobre estos temas y consultando los documentos oficiales de los respectivos ministerios de educación para ver qué estipulan al respecto, entre otras técnicas.

\section{4}

\section{CONCLUSIONES}

La retórica intercultural es una disciplina con medio siglo de trayectoria que ha modificado profundamente el discurrir de la disciplina del inglés como segunda lengua, aportando importantes perspectivas y nuevos métodos de investigación. Lamentablemente, se trata de un campo prácticamente reducido al ámbito científico anglosajón, por lo que sus avances no han sido extrapolados en la medida deseada a otras comunidades académicas, entre ellas al ELE. Debido a ello, consideramos que su metodología de investigación debe ser adaptada a este campo de la lingüística aplicada, puesto que ofrece numerosas líneas de investigación que enriquecerían enormemente al ELE. Como punto de partida se pueden tomar algunas de las diversas investigaciones ya realizadas que comparan el español con el inglés para mejorar esta última como segunda lengua o de las escasas llevadas a cabo concretamente en el ELE. Así, podrían conducirse estudios de análisis de géneros basados en corpus paralelos o de aprendientes. Especialmente relevante sería implementar investigaciones etnográficas que nos permitieran conocer mejor el porqué de determinados aspectos de la escritura. Por todo ello, concluimos que la metodología de la investigación de la retórica intercultural tiene mucho que aportar al ELE y es un camino por el que los especialistas deberían continuar en el futuro. 


\section{REFERENCIAS BIBLIOGRÁFICAS}

BALAŽIC BULC, Tatjana (2005) Connectors in Students’ Academic Writing in Two Closely Related Languages (on the Case of Slovene and Croatian Language). Universidad de Birmingham (ed.) Proceedings from the Corpus Linguistic Conference Series Vol. 1-2. Birmingham: Centre for corpus research. 30 de noviembre de 2015. http://www. birmingham.ac.uk/Documents/college-artslaw/corpus/conferencearchives/2005-journal/ContrastiveCorpusLinguistics/Connectorsinstudentsacademicwriting.doc

BECK, Cathy (2011) Appendix. U. Connor Intercultural Rhetoric in the Writing Classroom. Michigan: Universidad de Michigan, 105-106.

BELCHER, Diane/Gayle NELSON (eds.) (2013) Critical and Corpus-Based Approaches to Intercultural Rhetoric. Michigan: Universidad de Michigan.

CONNOR, Ulla (2004) Intercultural Rhetoric Research: Beyond Texts. Journal of English for Academic Purposes 3, 291-304.

CONNOR, Ulla (2011) Intercultural Rhetoric in the Writing Classroom. Michigan: Universidad de Michigan.

CONNOR, Ulla (2013) Corpus Linguistics in Intercultural Rhetoric. D. Belcher/G. Nelson (eds.) Critical and Corpus-Based Approaches to Intercultural Rhetoric. Michigan: Universidad de Michigan, 8-21.

CONNOR, Ulla/Ana I. MORENO (2005) Tertium comparationis: A vital component in contrastive research methodology. P. Bruthiaux/D. Atkinson/W. G. Eggington/W. Grabe/V. Ramanathan (eds.) Directions in Applied Linguistics: Essays in Honor of Robert B. Kaplan. Clevedon: Multilingual Matters, 153-164.

CONNOR, Ulla/William ROZYCKI (2013) ESP and Intercultural Rhetoric. B. Paltridge/ S. Starfield (eds.) The Handbook of English for Specific Purposes. Hoboken: John Wiley \& Sons, 427-443.

DEZA BLANCO, Pablo (2007) Los conectores en noticias de sucesos. Retórica contrastiva en textos de periodistas españoles y alumnos taiwaneses. Círculo de lingüistica aplicada a la comunicación. 28 de abril de 2015. http://pendientedemigracion.ucm. es/info/circulo/no30/deza.pdf.

ENKVIST, Nils E. (1997) Why We Need Contrastive Rhetoric. Alternation 4 (1), 188206.

ESCAMILLA, Ramon (2013) Discriminatory Discursive Strategies Used by the Japanese Mainstream News Media in Constructing the Identity of Resident Foreign Nationals: A Critical Discourse Analysis-Based Examination. D. Belcher/G. Nelson (eds.) Critical and Corpus-Based Approaches to Intercultural Rhetoric. Michigan: Universidad de Michigan, 72-96.

HEREDERO ZORZO, David (2016) El uso de marcadores discursivos en el género ensayo. Análisis de producciones escritas de estudiantes eslovenos desde la Retórica Contrastiva. Biblioteca virtual redELE 17. 
HEREDERO ZORZO, David (en prensa) De la retórica contrastiva a la retórica intercultural. Una nueva vuelta de tuerca al análisis contrastivo de la expresión escrita. Actas del III Congreso Internacional Nebrija en Lingüistica Aplicada a la Enseñanza de Lenguas.

JIMÉNEZ RAMÍREZ, Jorge (2010) Cómo aumentar la calidad de la producción escrita en la clase de E/LE: introducción a la retórica contrastiva mediante las diferencias ortotipográficas». ASELE (ed.) XXI congreso internacional de ASELE. Del texto a la enseñanza-aprendizaje del español L2-LE. Salamanca: ASELE, 265-273.

JOHANSSON, Stig (1998) On the role of corpora in cross-linguistic research. S. Johansson/S. Oksefjell (eds.) Corpora and Cross-linguistic Research: Theory, Method, and Case Studies. Amsterdam: Rodopi, 3-24.

KAPLAN, Robert B. (1966) Cultural Thought Patterns in Inter-Cultural Education. Language Learning 16, 1-20.

KAPLAN, Robert B./William GRABE (1996) Theory and Practice of Writing: An Applied Linguistic Perspective. Londres: Longman.

LOCASTRO, Virginia (2008) "Long Sentences and Floating Commas". Mexican Students' Rhetorical Practices and the Sociocultural Context. U. Connor/E. Nagelhout/W. V. Rozycki (eds.) Contrastive Rhetoric: Reaching to Intercultural Rhetoric. Filadelfia: John Benjamins B.V., 195-217.

MARTÍN MARTÍN, Pedro (2005) The Rhetoric of the Abstract in English and Spanish Scientific Discourse. A Cross-Cultural Genre Analytic Approach. Berna: Peter Lang. MORENO, Ana I. (1998) The explicit signalling of premise-conclusion sequences in research articles: A contrastive framework. Text 18, 545-585.

MORENO, Ana I. (2008) The Importance of Comparable Corpora in Cross-Cultural Studies. U. Connor/E. Nagelhout/W. V. Rozycki (eds.) Contrastive Rhetoric: Reaching to Intercultural Rhetoric. Filadelfia: John Benjamins B.V., 25-41.

MUR-DUEÑAS, Pilar (2016) Promotional strategies in academic writing: Statements of contribution in Spanish and ELF research articles. J. Pelclova/W. Lu (eds.) Persuasion in Public Discourse: Cognitive and Functional Perspectives. Amsterdam: John Benjamins.

PENAS IBÁÑEZ, María A./Raquel MARTÍN MARTÍN (2009) Traducción e Interculturalidad. Aspectos metodológicos teóricos y prácticos. Rabat: Universidad Mohamed $\mathrm{V}$ de Rabat.

PÉREZ-LLANTADA, Carmen (2008) Humans vs. Machines? A multi-perspective model for ESP discourse analysis in intercultural rhetoric research. ESP Across Cultures 5, 91-104.

SUÁREZ, Lorena/Ana I. MORENO (2008) The rhetorical structure of academic book reviews of literature: an English-Spanish cross-linguistic approach. U. Connor/E. Nagelhout/W. V. Rozycki (eds.) Contrastive Rhetoric: Reaching to Intercultural Rhetoric. Filadelfia: John Benjamins B.V., 147-168. 


\section{POVZETEK}

\section{Metodika raziskovanja medkulturne retorike, uporabljena za španščino kot tuji jezik}

Medkulturna retorika je disciplina, ki preučuje pisno izražanje med posamezniki iz različnih kultur. V anglosaksonskih znanstvenih krogih gre za izredno močno področje, še posebej v zvezi z angleščino kot drugim jezikom, kar pa ne velja za španščino kot tuji jezik. Medkulturna retorika je prispevala pomembne raziskovalne metode v uporabnem jezikoslovju. Zato smatramo, da lahko odpre zanimive perspektive tudi pri španščini kot tujem jeziku. $\mathrm{V}$ članki predstavljamo metodiko raziskovanja medkulturne retorike, ki temelji na uporabi različnih vrst korpusov za analizo besedilnih vrst, sledi osnovam tertium comparationis in uporablja tehnike etnografskih raziskav. Naš namen je pokazati uporabo te metodike za španščino kot tuji jezik in zasnovati možnosti za nadaljnje raziskave.

Ključne besede: raziskovalne metode, medkulturna retorika, španščina kot tuji jezik, tertium comparationis, etnografska raziskava

\section{ABSTRACT}

\section{The Methodology of Investigation of Intercultural Rhetoric applied to SFL}

Intercultural rhetoric is a discipline which studies written discourse among individuals from different cultures. It is a very strong field in the Anglo-Saxon scientific world, especially referring to English as a second language, but in Spanish as a foreign language it is not as prominent. Intercultural rhetoric has provided applied linguistics with important methods of investigation, thus applying this to SFL could introduce interesting new perspectives on the subject. In this paper, we present the methodology of investigation of intercultural rhetoric, which is based on the use of different types of corpora for analysing genders, and follows the precepts of tertium comparationis. In addition, it uses techniques of ethnographic investigation. The purpose of this paper is to show the applications of this methodology to SFL and to outline future investigations in the same field.

Key words: methods of investigation, intercultural rhetoric, SFL, tertium comparationis, etnographic investigation 


\section{RESUMEN}

\section{Metodología de investigación de la retórica intercultural aplicada al ELE}

La retórica intercultural es una disciplina que estudia el discurso escrito entre individuos procedentes de diferentes culturas. Dentro del mundo científico anglosajón es una corriente muy fuerte, concretamente en lo referente al inglés como segunda lengua, lo que no ocurre en el campo del español como lengua extranjera. La retórica intercultural ha aportado importantes métodos de investigación a la lingüística aplicada, por lo que la aplicación al ELE de esta metodología podría ofrecer perspectivas interesantes. En este trabajo se presenta la metodología de investigación de la retórica intercultural, basada en la utilización de diferentes tipos de corpus para el análisis de géneros y que sigue los preceptos del tertium comparationis, además de servirse de técnicas de investigación etnográficas. Ello se hace con el fin de mostrar las aplicaciones de esta metodología al ELE y esbozar las líneas de investigación abiertas al respecto.

Palabras clave: métodos de investigación, retórica intercultural, ELE, tertium comparationis, investigación etnográfica 